\title{
Dilatonic current-carrying cosmic strings
}

\author{
Patrick Peter* \\ Institut d'Astrophysique de Paris, GReCO, FRE 2435-CNRS, 98bis boulevard Arago, 75014 Paris, France \\ M. E. X. Guimarães ${ }^{\dagger}$ and V. C. de Andrade \\ Instituto de Física Teórica, Universidade Estadual Paulista, Rua Pamplona 145, 01405-900 São Paulo SP, Brazil
}

(Received 3 April 2003; published 17 June 2003)

\begin{abstract}
We investigate the nature of ordinary cosmic vortices in some scalar-tensor extensions of gravity. We find solutions for which the dilaton field condenses inside the vortex core. These solutions can be interpreted as raising the degeneracy between the eigenvalues of the effective stress-energy tensor, namely, the energy per unit length $U$ and the tension $T$, by picking a privileged spacelike or timelike coordinate direction; in the latter case, a phase frequency threshold occurs that is similar to what is found in ordinary neutral current-carrying cosmic strings. We find that the dilaton contribution for the equation of state, once averaged along the string worldsheet, vanishes, leading to an effective Nambu-Goto behavior of such a string network in cosmology, i.e. on very large scales. It is found also that on small scales, the energy per unit length and tension depend on the string internal coordinates in such a way as to permit the existence of centrifugally supported equilibrium configuration, also known as vortons, whose stability, depending on the very short distance (unknown) physics, can lead to catastrophic consequences on the evolution of the Universe.
\end{abstract}

DOI: 10.1103/PhysRevD.67.123509

PACS number(s): $98.80 . \mathrm{Cq}, 98.70 . \mathrm{Vc}$

\section{INTRODUCTION}

Most extensions of the standard model of particle physics predict that extra scalar fields, in addition to the ordinary Higgs field, whose experimental detection is still to be done, should exist in nature. At low energies (compared to the Planck scale), they appear to be classifiable into essentially two main categories; namely those which couple in a straightforward way to the other particle fields (as, e.g. grand unification breaking Higgs fields, supersymmetric partners of ordinary fermions or extra bosonic degrees of freedom coming from the Neveu-Schwarz sector of superstring theory [1]), and those whose most important coupling is to gravity, such as the dilaton, whose origin can be traced to the Ramond sector in the superstring context. Both kinds, coupled or decoupled, have been studied from different (and often disjoint) perspectives, and both have various cosmological and astrophysically observable consequences; these terms permit us, for instance, to obtain fully nonsingular cosmologies [2]. In particular, scalar-tensor theories of gravity [3] may provide a natural solution to the problem of terminating inflation [4], whilst grand unified theory (GUT) scalars, being symmetry breakers, may lead to the formation of topological defects [5], of which only cosmic strings are viable candidates from the point of view of cosmology.

Among these theories, some predict both kinds of fields. As a result, one expects that cosmic strings could exist whose coupling to gravity would be altered by inclusion of dilaton effects. In Ref. [6], a local cosmic string solution was considered in the framework of low energy string theory

\footnotetext{
*Electronic address: peter@iap.fr

${ }^{\dagger}$ On leave from Universidade de Brasília, Departamento de Matemática. Electronic address: emilia@ift.unesp.br

‡Electronic address: andrade@ift.unesp.br
}

which is reminiscent of the scalar-tensor theories of gravity [3]. Indeed, a massless dilaton is shown to obey a least coupling principle [7], e.g. to decouple from matter by cosmological attraction in much the same way as the generic attractor mechanism of the scalar-tensor theories of gravity [8]. It was found [6] then that the metric around a cosmic string in the framework of scalar-tensor gravity is of the Taub-Kasner type [9] so that the particle and light propagation resembles that around a wiggly cosmic string in ordinary general relativity [10], although the effect was expected to be one order of magnitude stronger.

Here, we want to point out another effect, namely that the dilaton field may behave as a winding phase along the string, thereby generating a neutral current kind of effect by raising the degeneracy between the eigenvalues of the stress-energy tensor. From the point of view of purely gravitational physics, this seems utterly negligible as the metric would hardly be affected $[11,12]$ by such a current (it gives again a Kasner-like metric, up to second order corrections).

The most noticeable consequence of a current-like effect is [13-15] to modify the internal dynamics of cosmic strings in such a way that new states are reachable. Indeed, the breaking of the Lorentz boost invariance along the worldsheet allows rotating equilibrium configurations, called vortons, which, if they are stable, can overclose the universe, thereby leading to a catastrophe for the theory that predicts them [16]. Finally, inclusion of such an internal structure could drastically change the predictions of a cosmic string model [17] in the microwave background anisotropies [18]. Here we show that the long-range effect on a cosmologically relevant network of strings is vanishing on average, but that vorton-like states can be reached by microscopically small loops.

In what follows, after having set the relevant gravitational theory and notations, we derive the corresponding field equations in Sec. II. We then set the vortex (Abelian Higgs) 
model which we develop in flat space in Sec. III with the aim of using it as a source for the gravitational effects. We then move on to obtain, in this framework, the general solution for the dilaton field in the Einstein frame (Sec. IV). We apply this solution to derive the effective stress-energy tensor (Sec. V) of the string, as seen from a Jordan-Fierz frame observer. We show that this stress-energy tensor has very particular features that can be interpreted by saying that a network of such string will evolve on cosmologically relevant scales as a usual network [19] of Nambu-Goto strings [20], but might lead to the formation of equilibrium vorton states [16] whose density, scaling as matter, could overclose the Universe in the case in which they are stable, an issue which is yet unresolved, depending on the small distance physics. Section VI summarizes our findings and discusses the relevant cosmological conclusions.

\section{GRAVITATIONAL FRAMEWORK}

We start with the gravitation action in the Jordan-Fierz frame (also known as the "string" frame, a nomenclature we shall not use in order to avoid the possible risk of confusion with the strings of the cosmic kind we consider below), namely

$$
\begin{aligned}
\mathcal{S}_{\mathrm{JF}}= & \frac{1}{16 \pi} \int \mathrm{d}^{4} x \sqrt{-\tilde{g}}\left[\widetilde{R} \widetilde{\Phi}-\frac{\omega(\widetilde{\Phi})}{\widetilde{\Phi}} \partial^{\mu} \widetilde{\Phi} \partial_{\mu} \widetilde{\Phi}\right] \\
& +\mathcal{S}_{\mathrm{m}}\left[\Psi_{\mathrm{m}}(x), \tilde{g}_{\mu \nu}(x)\right],
\end{aligned}
$$

where $\tilde{g}_{\mu \nu}$ is the physical metric which contains both scalar and tensor degrees of freedom, $\widetilde{R}$ is the curvature scalar associated with it, and $\mathcal{S}_{\mathrm{m}}$ is the action for general matter fields $\Psi_{\mathrm{m}}$ which, at this point, is left arbitrary. The metric signature is assumed to be $(+,-,-,-)$.

By varying the action (1) with respect to the metric $\tilde{g}_{\mu \nu}$ and to the scalar field $\widetilde{\Phi}$ we obtain the "modified" Einstein

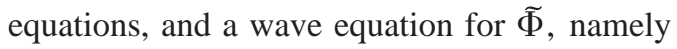

$$
\begin{aligned}
\widetilde{G}_{\mu \nu}= & \frac{8 \pi}{\widetilde{\Phi}} \widetilde{T}_{\mu \nu}+\frac{1}{\widetilde{\Phi}}\left(\widetilde{\nabla}_{\nu} \widetilde{\Phi}_{, \mu}-\widetilde{g}_{\mu \nu} \square \widetilde{\Phi}\right)+\frac{\omega(\widetilde{\Phi})}{\widetilde{\Phi}^{2}} \\
& \times\left(\partial_{\mu} \widetilde{\Phi} \partial_{\nu} \widetilde{\Phi}-\frac{1}{2} \widetilde{g}_{\mu \nu} \partial_{\alpha} \widetilde{\Phi} \partial^{\alpha} \widetilde{\Phi}\right), \\
\widetilde{\square} \widetilde{\Phi}= & \frac{1}{2 \omega(\widetilde{\Phi})+3}\left(8 \pi \widetilde{T}-\frac{\mathrm{d} \omega}{\mathrm{d} \widetilde{\Phi}} \partial_{\mu} \widetilde{\Phi} \partial^{\mu} \widetilde{\Phi}\right), \\
\widetilde{\nabla}_{\mu} \widetilde{T}_{\nu}^{\mu}= & 0,
\end{aligned}
$$

where a tilde over a differential operator means it is built out of the Jordan-Fierz metric $\tilde{g}_{\mu \nu}$,

$$
\widetilde{G}_{\mu \nu}=\widetilde{R}_{\mu \nu}-\frac{1}{2} \widetilde{g}_{\mu \nu} \widetilde{R}
$$

is accordingly the Einstein tensor in the Jordan-Fierz frame, and

$$
\widetilde{T}_{\mu \nu} \equiv \frac{2}{\sqrt{-\tilde{g}}} \frac{\delta \mathcal{S}_{\mathrm{m}}}{\delta \widetilde{g}^{\mu \nu}}
$$

is the energy-momentum tensor of the matter content and $\widetilde{T}$ $\equiv \widetilde{T}^{\mu}{ }_{\mu}$ is its trace. Clearly, if $\widetilde{T}$ vanishes and $\widetilde{\Phi}$ is a constant, Eq. (2) reduces to the usual set of Einstein field equations if we identify the inverse of the scalar field with the Newton constant, i.e. $G_{\mathrm{N}}=1 / \widetilde{\Phi}$. Hence, any exact solution of Einstein equations with a trace-free matter source will also be a particular exact solution of the scalar-field with $\widetilde{\Phi}$ constant. Of course, this particular solution will not be, except in very special situations, the general solution for the matter content [21].

Let us rewrite the action given by Eq. (1) in terms of the Einstein (conformal) frame in which the kinetic terms of tensor and scalar degrees of freedom do not mix, i.e.

$$
\begin{aligned}
\mathcal{S}_{\mathrm{E}}= & \frac{1}{16 \pi G^{*}} \int \mathrm{d}^{4} x \sqrt{-g}\left(R-2 g^{\mu \nu} \partial_{\mu} \phi \partial_{\nu} \phi\right) \\
& +\mathcal{S}_{\mathrm{m}}\left[\Psi_{\mathrm{m}}, A^{2}(\phi) g_{\mu \nu}\right],
\end{aligned}
$$

where $g_{\mu \nu}$ is a pure rank-2 metric tensor, $R$ is the curvature scalar associated to it, and $G^{*}$ the bare gravitational constant.

As is well known, the action given by Eq. (7) is obtained from that of Eq. (1) by means of a conformal transformation

$$
\tilde{g}_{\mu \nu}=A^{2}(\phi) g_{\mu \nu},
$$

provided the scalar field functions $\phi$ and $\Phi$ are related through

$$
G^{*} A^{2}(\phi)=\frac{1}{\widetilde{\Phi}}
$$

and

$$
\frac{\mathrm{d} \ln A(\phi)}{\mathrm{d} \phi}=\frac{1}{\sqrt{2 \omega(\widetilde{\Phi})+3}} \equiv \alpha(\phi),
$$

(thus defining the function $\alpha$ ) which can be interpreted as the (field-dependent) coupling strength between matter and scalar field.

In the conformal frame, Eqs. (2) and (3) are written in a more convenient form

$$
G_{\mu \nu}=2 \partial_{\mu} \phi \partial_{\nu} \phi-g_{\mu \nu} g^{\alpha \beta} \partial_{\alpha} \phi \partial_{\beta} \phi+8 \pi G^{*} T_{\mu \nu},
$$

for the gravitational part, and

$$
\square \phi=-4 \pi G^{*} \alpha(\phi) T,
$$

for the dilatonic part, where now the matter stress-energy tensor $T_{\mu \nu}$ is obtained from 


$$
T_{\mu \nu} \equiv \frac{2}{\sqrt{-g}} \frac{\delta \mathcal{S}_{\mathrm{m}}}{\delta g^{\mu \nu}},
$$

which in this new frame is no longer conserved unless the dilaton is constant, i.e. $\nabla_{\mu} T_{\nu}^{\mu}=\alpha(\phi) T \nabla_{\nu} \phi$. The Einstein frame Einstein tensor $G_{\mu \nu}$ appearing in Eq. (11) is defined in the same was as Eq. (5) without the tildes. From Eq. (8), we can easily relate quantities from both frames in the following way:

$$
\widetilde{T}_{\mu \nu}=A^{-2}(\phi) T_{\mu \nu},
$$

which also implies $\widetilde{T}^{\mu}{ }_{\nu}=A^{-4} T^{\mu}{ }_{\nu}$ and $\widetilde{T}^{\mu \nu}=A^{-6} T^{\mu \nu}$. For the sake of generality, we choose to leave $A(\phi)$ as an arbitrary function of the scalar field.

Let us now turn to the cosmic string source terms and consider the microscopic field theory out of which vortices stem.

\section{VORTEX FIELD MODEL}

We shall now consider the underlying field model that gives birth to cosmic strings. It consists in a complex scalar Higgs field $\varphi$, coupled to a gauge vector $B_{\mu}$. Both fields are, as discussed above, minimally coupled to gravity so that the matter action we shall deal with is expressible as

$$
\mathcal{S}_{\mathrm{m}}=\int \mathrm{d}^{4} x \sqrt{-\tilde{g}}\left(\frac{1}{2}|D \varphi|^{2}-\frac{1}{4} H_{\mu \nu} H^{\mu \nu}-V\right),
$$

where the $\mathrm{U}(1)$ covariant derivative is $D_{\mu} \equiv \partial_{\mu}+i q B_{\mu}$, the "Faraday"-like tensor $H_{\mu \nu} \equiv \partial_{\mu} B_{\nu}-\partial_{\nu} B_{\mu}$, and the Higgs potential reads $V(\varphi)=\lambda\left(\varphi^{\star} \varphi-\eta^{2}\right)^{2}$; all indices are raised and lowered by means of the metric $\tilde{g}$.

We shall from now on consider the zeroth order approximation for the background fields. This means we are interested in the string as a source for the gravitational and dilaton fields. As a result, in order to derive the relevant stressenergy tensor, we demand that the Einstein-frame metric be that of Minkowski, while the dilaton assumes a constant value, i.e.

$$
\widetilde{\Phi}_{(0)}=\widetilde{\Phi}_{0} \Rightarrow \phi_{(0)}=\phi_{0},
$$

so that, at this order, gravity is described by general relativity in both frames and the Jordan-Fierz metric can be taken, in a cylindrical coordinate system $(t, z, r, \theta)$, as

$$
\tilde{g}_{(0)}^{\mu \nu}=A_{0}^{-2} \eta^{\mu \nu}=A_{0}^{-2} \operatorname{Diag}\left(1,-1,-1,-\frac{1}{r^{2}}\right),
$$

i.e. again the Minkowski metric [up to a constant scaling factor $\left.A_{0} \equiv A\left(\phi_{0}\right)\right]$. The usual Newton constant is then $G_{\mathrm{N}}$ $=\widetilde{\Phi}_{0}^{-1}=G^{*} A_{0}^{2}$. Note that in Eq. (17) we have inserted the (constant) conformal factor in the Jordan-Fierz metric: this is just for further convenience since we will be mostly working in the Einstein frame in which this extra factor will then be absent.

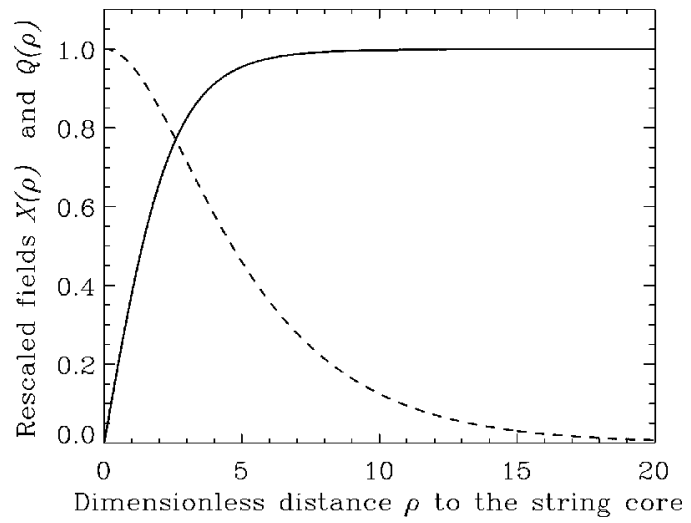

FIG. 1. Numerical solutions for the rescaled Higgs (full line) and gauge (dashed line) fields around a vortex in a Minkowski background with $A_{0}=1$. Adapted from Ref. [15].

There exist static vortex configurations that are solutions of the Euler-Lagrange equations derivable from the action given by Eq. (15). Such a configuration, for a string along the $z$ axis, has the form [22]

$$
\varphi=h(r) \mathrm{e}^{i n \theta} \text {, and } B_{\mu}=\frac{1}{q}[Q(r)-n] \delta_{\mu}^{\theta},
$$

where the functions $h$ and $Q$ depend on the radial distance to the string core $r$ only. In what follows, for the sake of definiteness, we shall also assume that the underlying parameters in the matter action are such that only the vortices with winding number $n=1$ are stable and we shall therefore concentrate our attention on these configurations. Note however that this requirement will not modify our conclusions, since what is presented here is merely an existence proof that only relies on the presence of the defect itself.

Using a prime to denote differentiation with respect to the radial distance $r$, the field equations derivable from the action (15) are

$$
h^{\prime \prime}+\frac{h^{\prime}}{r}=h\left[\frac{Q^{2}}{r^{2}}+4 \lambda A_{0}^{2}\left(h^{2}-\eta^{2}\right)\right]
$$

and

$$
Q^{\prime \prime}-\frac{Q^{\prime}}{r}=q^{2} Q A_{0}^{2} h^{2},
$$

and the boundary conditions for these fields to describe a vortex line read

$$
\begin{gathered}
h(0)=0, \quad Q(0)=1, \\
\lim _{r \rightarrow \infty} h(r)=\eta, \quad \lim _{r \rightarrow \infty} Q(r)=0 .
\end{gathered}
$$

The field equations (19) and (20), together with the conditions (21) are usually solved numerically; an example of such a solution is shown on Fig. 1, adapted from Ref. [15]. On the figure are shown the dimensionless quantities 


$$
X \equiv \frac{h}{\eta}
$$

and $Q(\rho)$ as functions of the rescaled (dimensionless) distance to the string core

$$
\rho \equiv \frac{r}{r_{\mathrm{h}}}, \text { where } r_{\mathrm{h}} \equiv \lambda^{-1 / 2} \eta^{-1}
$$

is the Compton wavelength of the Higgs field. Such a generic configuration acts as a source for the dilaton field.

In order to derive the internal string solution for the dilaton field in which we are interested, we need to first obtain the stress-energy tensor (13), namely

$$
\begin{aligned}
\widetilde{T}_{\mu \nu}= & \frac{1}{2}\left[\left(D_{\mu} \varphi\right)^{\dagger} D_{\nu} \varphi+\left(D_{\nu} \varphi\right)^{\dagger} D_{\mu} \varphi\right]-\frac{1}{2} \widetilde{g}_{\mu \nu}|D \varphi|^{2} \\
& +\widetilde{g}_{\mu \nu} V(\varphi)+\frac{1}{4} \widetilde{g}_{\mu \nu} H^{2}-\widetilde{g}^{\alpha \beta} H_{\mu \alpha} H_{\nu \beta},
\end{aligned}
$$

and, setting $V \equiv V(h)=\lambda\left(h^{2}-\eta^{2}\right)^{2}$, this yields the following components:

$$
\begin{aligned}
& \widetilde{T}_{z}^{z}=V+\frac{A_{0}^{-2}}{2}\left[h^{\prime 2}+\frac{1}{r^{2}}\left(h^{2} Q^{2}+\frac{Q^{\prime 2}}{q^{2} A_{0}^{2}}\right)\right], \\
& \widetilde{T}_{r}^{r}=V-\frac{A_{0}^{-2}}{2}\left[h^{\prime 2}-\frac{1}{r^{2}}\left(h^{2} Q^{2}-\frac{Q^{\prime 2}}{q^{2} A_{0}^{2}}\right)\right], \\
& \widetilde{T}_{\theta}^{\theta}=V+\frac{A_{0}^{-2}}{2}\left[h^{\prime 2}-\frac{1}{r^{2}}\left(h^{2} Q^{2}+\frac{Q^{\prime 2}}{q^{2} A_{0}^{2}}\right)\right],
\end{aligned}
$$

and $\widetilde{T}_{t}^{t}=\widetilde{T}_{z}^{z}$. This zeroth order stress-energy tensor should in principle be used as a source for the modified Einstein equations.

It can be noted that, as is clear from Eqs. (19) and (20) as well as Eqs. (25)-(27), the normalization $A_{0}$ of the dilaton function $A(\phi)$ can be modified at will provided one performs simultaneously a redefinition of the coupling constants $\lambda$ and $q$ through $\bar{\lambda}=A_{0}^{2} \lambda$ and $\bar{q}=A_{0} q$. In fact, this normalization turns out to be completely irrelevant for the vortex configurations since all the properties of such vortices only depend on the ratio [15] $\lambda / q^{2}=\bar{\lambda} / \bar{q}^{2}$. This stems from the fact that $A_{0}^{2} \widetilde{T}^{\mu}{ }_{\nu}$ can be expressed in terms of $\bar{\lambda}$ and $\bar{q}$ only, so the only effect is a normalization one. It is therefore possible in principle to set $A_{0}=1$, a convention within which the metrics in either the Jordan-Fierz or the Einstein frame are exactly equal. In order to distinguish between these frame, we shall however not adopt this convention, unless stated otherwise.

\section{FIRST ORDER DILATON SOLUTION}

We now switch to the Einstein frame. The stress-energy tensor just derived then provides the new frame one through the relation (14), so that the trace needed in Eq. (12) thus

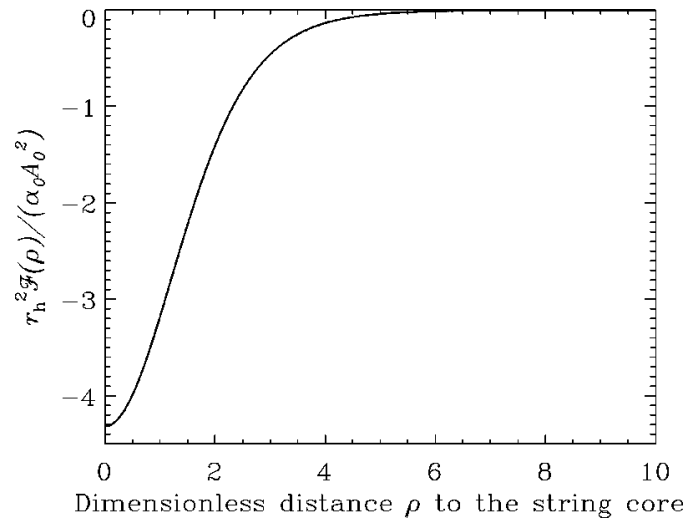

FIG. 2. Source function for the dilaton in Eq. (29) with the vortex solution of Fig. 1.

takes the form $T=A^{4} \widetilde{T}$. Inserting Eqs. (25)-(27) into Eq. (12), we obtain the dynamical equation, up to first order in the gravitational constant, for the dilaton as

$$
\phi=-4 \pi G^{*} \alpha\left(\phi_{0}\right) A_{0}^{2}\left[h^{\prime 2}+\frac{h^{2} Q^{2}}{r^{2}}+4 \lambda A_{0}^{2}\left(h^{2}-\eta^{2}\right)^{2}\right],
$$

which may be expressed as

$$
\square \phi=\epsilon \mathcal{F}(r),
$$

where we have set $\alpha_{0} \equiv \alpha\left(\phi_{0}\right)$. The function $\mathcal{F}$ on the right hand side of Eq. (29) is given, in terms of the dimensionless quantities, by [see Eq. (22) and below]

$$
\mathcal{F}=-\frac{\alpha_{0} A_{0}^{2}}{r_{\mathrm{h}}^{2}}\left[\left(\frac{\mathrm{d} X}{\mathrm{~d} \rho}\right)^{2}+\frac{X^{2} Q^{2}}{\rho^{2}}+4 A_{0}^{2}\left(X^{2}-1\right)^{2}\right],
$$

and is exhibited in Fig. 2.

In Eq. (29), we have emphasized the constant combination

$$
\epsilon \equiv 4 \pi G^{*} \eta^{2} \sim 4 \pi\left(\frac{\eta}{M_{\mathrm{P}}}\right)^{2}
$$

which will be used in what follows as a small expansion parameter. Indeed, even for the highest possible energy phase transition leading to cosmic strings compatible with cosmological data [23], i.e. the GUT scale, the quantity $\eta$ is of order $10^{15}-10^{16} \mathrm{GeV}$, which is at most three orders of magnitude smaller than the Planck scale $M_{\mathrm{P}} \equiv G_{\mathrm{N}}^{-1 / 2}$ so that one has $\epsilon \lesssim 10^{-5}$.

Let us now expand all the fields involved in terms of the small parameter $\epsilon$. In what follows, we shall concentrate on the dilaton field, because the solution of the Einstein equations (11) for the metric has already been obtained in the cosmic string case [6]. In this reference, it had been found that the external metric, far from the string core, was of the Kasner-like form 


$$
\mathrm{d} s^{2}=\left(\frac{r}{r_{0}}\right)^{k}\left(\mathrm{~d} t^{2}-\mathrm{d} z^{2}\right)-\mathrm{d} r^{2}-\Gamma r^{2}\left(\frac{r}{r_{0}}\right)^{-2 k} \mathrm{~d} \theta^{2},
$$

with $\Gamma$ and $k$ two arbitrary constants, and that it could be matched with the interior solution provided $k=4 / 3$ or $k=0$ in the presence of the string.

From Fig. 2, it is clear that there exists a distance $r_{0}$ such that for $r \geqslant r_{0}$, the source function is approximately vanishing, so that the exterior solution for the dilaton should satisfy

$$
\square \phi_{\mathrm{ext}}=0 .
$$

Therefore, the dilaton field, in vacuum $\left(T_{\mu \nu}=0\right)$, assumes the general form [6]

$$
\phi_{\mathrm{ext}}=\phi_{0}+\kappa \ln \left(\frac{r}{r_{0}}\right)
$$

where $\phi_{0}$ and $\kappa$ are constant, the latter being determined by a matching with the internal solution, while the former gives the strength of gravitational coupling.

In the absence of a string, one would have $\kappa=0$, as demanded also by the requirement that the Ricci tensor be regular [6]. This solution, as it turns out [6], is valid, to first order in the weak field approximation, both inside and outside the string. We will thus use this solution to derive the string structure itself, and show, for self-consistency, that a modification of the dilaton solution with respect to Ref. [6] does not modify this metric (again, at least to first order in $\epsilon$ ). The mild (logarithmic) divergence observed in Eq. (34) for the dilaton far from the string stems from the infinite string approximation we are making use of, and can easily be accounted for by introducing a long range cutoff such as, e.g. the curvature radius of the string, or the interstring distance in a cosmological network. We shall see later that it can also be altogether cancelled once the source term is taken into account [see the discussion below Eq. (43)].

It is interesting to note here that the solution (34) for the dilaton actually also diverges as $r \rightarrow 0$, implying a breakdown of the underlying four-dimensional effective field theory. This is similar to the situation encountered when the axion field is taken in consideration in cosmic strings formed at the symmetry breaking of the pseudo-anomalous $\mathrm{U}(1)$ that characterizes [24] most cases of superstring compactification [25], indicating that topological defect cores might be objects of comparable theoretical interest as black hole or cosmological singularities in that they probably require a full knowledge of the nonlinear theory to be properly understood. In what follows, we shall assume a short-range cutoff for the dilaton, expected to be of order $M_{\mathrm{P}}^{-1}$, and subsequently neglect distances shorter than the Planck length; inclusion of this cutoff scale merely renormalizes the string energy per unit length and tension by factors of order unity [24] that are irrelevant to the following discussion. Another implication of this divergence is that some cosmologically interesting effects, such as formation of wakes by dilatonic strings, may break down due to the logarithmic divergence of this solution [26].

Assuming the dilaton to behave as

$$
\phi=\phi_{0}+\epsilon \phi_{1}
$$

where, as before, $\phi_{0}$ is the constant dilaton value in the absence of string, and $\phi_{1}$ depends on the radial coordinate $r$ as well as the string coordinates $z$ and $t$ (we assume rotational symmetry so that $\partial_{\theta} \phi_{1}=0$ ), Eq. (29) inside the string, up to first order in $\epsilon$, becomes

$$
\left(\ddot{\phi}_{1}-\overline{\bar{\phi}}_{1}\right)-\frac{1}{r} \frac{\partial}{\partial r}\left(r \frac{\partial \phi_{1}}{\partial r}\right)=\mathcal{F}(r),
$$

where a dot and a bar respectively stand for derivations with respect to the coordinates $t$ and $z$.

As we want to match the solution of Eqs. (35) and (36) with the already derived solution (34), we seek the following form:

$$
\phi_{1}(t, r, z)=\chi(r)+f(r) \psi(z, t),
$$

where the function $f(r)$ is required to vanish asymptotically far from the defect, i.e. in practice for $r \geqslant r_{0}$, in order to ensure that the corresponding effect is localized into the worldsheet only. Note that the $z$ and $t$ dependence of the dilaton in Eq. (37) is not incompatible with the assumption of time-independence and cylindrical symmetry for the metric at the first order in $\epsilon$. Indeed, as can readily be seen in Eqs. (11) and (35), the leading contribution in the metric of the correction $\phi_{1}$ is second order in $\epsilon$. With such a tentative solution, Eq. (36) reduces to

$$
(\ddot{\psi}-\overline{\bar{\psi}}) f-\frac{1}{r} \frac{\mathrm{d}}{\mathrm{d} r}\left(r \frac{\mathrm{d} \chi}{\mathrm{d} r}\right)-\frac{1}{r} \frac{\mathrm{d}}{\mathrm{d} r}\left(r \frac{\mathrm{d} f}{\mathrm{~d} r}\right) \psi=\mathcal{F}(r) .
$$

In order for our solution to be valid regardless of the behavior of $\psi(z, t)$, i.e. including the case $\psi=0$, we demand that the function $\mathcal{F}$ sources only the pure radial component of the dilaton, i.e. we impose

$$
\chi^{\prime \prime}+\frac{1}{r} \chi^{\prime}=\mathcal{F}(r)
$$

which implies

$$
\chi=A+B \ln \left(\frac{r}{r_{0}}\right)+\chi_{\mathrm{s}}
$$

where $A$ and $B$ are two arbitrary constants and the special solution

$$
\chi_{\mathrm{S}}=\int_{r_{1}}^{r} \frac{\mathrm{d} \tilde{r}}{\tilde{r}} \int_{r_{2}}^{\tilde{r}} \mathcal{F}(\bar{r}) \bar{r} \mathrm{~d} \bar{r}
$$

depends a priori on the two constants $r_{1}$ and $r_{2}$. Note however that $r_{1}$ and $r_{2}$ have no physical influence and can be chosen at will. In particular, it is convenient to set $r_{1}=r_{2}$ $=0$, so that, with $\mathcal{F} \sim \mathcal{F}_{0}+\mathcal{F}_{2} r^{2}$, which is the short distance behavior of $\mathcal{F}$ (see Fig. 2), one gets 


$$
\chi \sim A+B \ln \left(\frac{r}{r_{0}}\right)+\frac{\mathcal{F}_{0}}{4} r^{2}+\frac{\mathcal{F}_{2}}{9} r^{3}+\cdots,
$$

close to the string core, i.e. in the limit $r \rightarrow 0$. The special solution $\chi_{\mathrm{S}}$ cannot alleviate the divergence of the dilaton near the string core.

On the other hand, Eq. (41) can also be used to match the exterior solution (34) to the interior solution. In particular, it is interesting to note that for large distances, and because the function $\mathcal{F}$ vanishes exponentially fast, ${ }^{1}$ one has, for large values of $r$, e.g. $r>r_{\infty}$ with $r_{\infty}$ far away from the string core,

$$
\begin{aligned}
\chi_{\mathrm{S}} & =\int_{0}^{r_{\infty}} \frac{\mathrm{d} \tilde{r}}{\tilde{r}} \int_{0}^{\tilde{r}} \mathcal{F}(\bar{r}) \bar{r} \mathrm{~d} \bar{r}+\int_{r_{\infty}}^{r} \frac{\mathrm{d} \tilde{r}}{\tilde{r}} \int_{0}^{\tilde{r}} \mathcal{F}(\bar{r}) \bar{r} \mathrm{~d} \bar{r} \\
& \simeq \text { f.p. }+\left[\int_{0}^{\infty} \mathcal{F}(\tilde{r}) \tilde{r} \mathrm{~d} \tilde{r}\right] \ln \left(\frac{r}{r_{\infty}}\right),
\end{aligned}
$$

where "f.p." stands for the finite part of the above relation. Because the constant $B$ in Eq. (40) is, at this stage, arbitrary, it can be chosen to exactly compensate for the asymptotic logarithmic divergence in $\chi_{\mathrm{s}}$, in such a way that the exterior solution for the dilaton can be consistently imposed to be a constant, i.e. $\kappa=0$ in Eq. (34). As opposed to any other choice, this one leads to a finite amount of energy. This is reminiscent of what happens around the vortices studied in Ref. [24], whose coupling with the axion made them local even though they were initially global.

Now, returning to Eq. (38) in which we insert the solution for $\chi$ and separating, we obtain

$$
\frac{1}{\psi}(\ddot{\psi}-\overline{\bar{\psi}})=\frac{1}{f}\left(f^{\prime \prime}+\frac{1}{r} f^{\prime}\right)=w,
$$

with $w$ a constant. Therefore, we have the following set of equations:

$$
\ddot{\psi}-\overline{\bar{\psi}}=w \psi
$$

and

$$
f^{\prime \prime}+\frac{1}{r} f^{\prime}=w f
$$

for $f$ satisfying the boundary condition $\lim _{r \rightarrow \infty} f(r)=0$. The arbitrary constant $w$ can assume $a$ priori both positive and negative values, so we will inspect both cases in turn later, but from now on let us consider Eq. (45) for the phase modulation $\psi$ depending on the variables $(t, z)$, and seek a solution of the form

$$
\psi(t, z)=\psi(k z-\omega t) \equiv \psi(u),
$$

which gives

\footnotetext{
${ }^{1}$ This can be seen through a careful examination of the asymptotic behaviors of the various fields involved, as derived, e.g. in Ref. [15].
}

$$
\left(\omega^{2}-k^{2}\right) \frac{\mathrm{d}^{2} \psi}{\mathrm{d} u^{2}}=w \psi
$$

An overall rescaling of the coordinates being always possible, we can without lack of generality assume that $w=$ $\pm\left(\omega^{2}-k^{2}\right)$. The positive sign choice however leads to exponentially growing or decaying solutions in the variable $u$ which are either unbounded or vanishing, hence physically irrelevant. We are thus led to impose the negative sign, namely

$$
w=k^{2}-\omega^{2},
$$

and the solution for the dilaton

$$
\psi=\psi_{\mathrm{s}} \sin (k z-\omega t)+\psi_{\mathrm{c}} \cos (k z-\omega t) .
$$

It is worth noting that if $w \geqslant 0$ (respectively $w \leqslant 0$ ), the variable $u$ defines a new spacelike (resp. timelike) coordinate. In order to simplify the following calculations, we shall perform a Lorentz boost along the string such that if $w \geqslant 0$ (respectively $w \leqslant 0$ ), the new time and space coordinates $t^{\prime}$ and $z^{\prime}$ and the corresponding new constants $\omega^{\prime}$ and $k^{\prime}$ are such that $u=k^{\prime} z^{\prime}$ (resp. $\left.u=-\omega^{\prime} t^{\prime}\right)$ and $w=k^{\prime 2}$ (resp. $w=$ $\left.-\omega^{\prime 2}\right)$. Assuming this new frame from now on, we will then drop the primes as there is no risk of confusion.

Consider first the case for which $w=-\omega^{2}$, i.e. a negative constant. It can be seen that Eq. (46) then becomes the Bessel equation of order zero, with general solution [27]

$$
f(r)=f_{J} J_{0}(\omega r)+f_{Y} Y_{0}(\omega r)
$$

with a priori arbitrary numerical coefficients $f_{J}$ and $f_{Y}, J_{0}$ and $Y_{0}$ being respectively Bessel functions of the first and second kind. The boundary condition that $f$ should vanish asymptotically is not enough to impose any condition on the choice of the constants; even the fact that $Y_{0}$ diverges near the axis does not lead to any new constraint since $Y_{0}(\omega r)$ $\propto \ln (\omega r)$, i.e. a divergence similar to that already observed for the radial part $\chi(r)$, for which a cutoff needs to be imposed at the Planck scale. However, as we shall show later, there are other constraints stemming from the requirement that the eigenvalues of the energy-momentum tensor, once integrated in the directions transverse to the string, be finite.

Let now $w$ be a positive constant, i.e. $w=k^{2}$. Equation (46) is in this case the modified Bessel function of order zero, with solution

$$
f(r)=f_{I} I_{0}(k r)+f_{K} K_{0}(k r) .
$$

Here again, the constants $f_{I}$ and $f_{K}$ are a priori arbitrary and must be designed in such a way as to match the exterior solution. It is clear however that since we demand the dilaton first order correction to vanish asymptotically, we must impose $f_{I}=0$ since $I_{0}$ is exponentially divergent for large arguments. Note that here as well as in the previous case (51), the solution involves a logarithmic divergence reminiscent of the behavior given by Eq. (34), whose significance is discussed underneath that equation. 
Equations (51) and (52), together with Eq. (50), appear to completely determine the space-time behavior of the dilaton field in all possible situations. We now turn to the consequences that this solution produces in the effective stressenergy tensor.

\section{EFFECTIVE STRESS-ENERGY TENSOR}

We now consider the effective stress-energy tensor $\widetilde{T}_{\text {(eff) }}^{\mu \nu}$ that is seen by an observer wishing to describe the string behavior in the framework of Einstein general relativity. This means $\widetilde{T}_{\text {(eff) }}^{\mu \nu}$ is given by assuming Eq. (2) takes the form

$$
\widetilde{R}_{\mu \nu}-\frac{1}{2} \widetilde{g}_{\mu \nu} \widetilde{R}=8 \pi G_{\mathrm{N}} \widetilde{T}_{\mu \nu}^{\text {(eff) }},
$$

a relation that we will use later to identify the effective energy per unit length and tension of the string to first order in $\epsilon$. In order to achieve this goal, let us remark that Eq. (9) implies that

$$
\frac{1}{\widetilde{\Phi}} \sim G^{*} A_{0}^{2}\left(1+2 \epsilon \alpha_{0} \phi_{1}\right)
$$

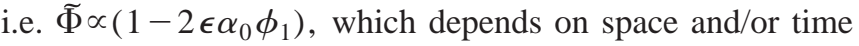
coordinates only through the first order dilaton field $\phi_{1}$. Plugging this form back into Eq. (53), keeping in mind that $G_{\mathrm{N}} \widetilde{T}_{\text {(eff) }}^{\mu \nu}$ is already a first order quantity and that $\partial_{\mu} \widetilde{\Phi}=$ $-2 \alpha_{0} \epsilon \partial_{\mu} \phi_{1} / G_{\mathrm{N}}$, we find that, to first order, the effective stress-energy tensor we are seeking reads

$$
8 \pi \widetilde{T}_{\mu \nu}^{(\mathrm{eff})}=8 \pi \widetilde{T}_{\mu \nu}^{(0)}+\left(\partial_{\mu} \partial_{\nu}-\Gamma_{\mu \nu}^{\alpha} \partial_{\alpha}-\eta_{\mu \nu} \square\right) \widetilde{\Phi}+\mathcal{O}_{\mu \nu}^{(2)},
$$

where $\widetilde{T}_{\mu \nu}^{(0)}$ is the zeroth order stress-energy tensor given by Eqs. (25)-(27) and $\mathcal{O}_{\mu \nu}^{(2)}$ contains only terms proportional to $\epsilon^{2}$.

In order to determine the influence of the dilaton field on the string dynamics, let us first recall the relevant pieces of formalism needed to describe it from the macroscopic point of view [14]. We shall consider our string to be describable by means of a surface action and accordingly integrate the effective stress-energy tensor over the transverse degrees of freedom, i.e.

$$
\widetilde{T}_{\|}^{\mu \nu} \equiv \int \widetilde{T}_{\text {(eff) }}^{\mu \nu} \mathrm{d}^{2} x^{\perp}
$$

where $\mathrm{d}^{2} x^{\perp}$ accounts for the transverse measure around the string. To the required zeroth order (since the integrand $\widetilde{T}_{\mu \nu}^{\text {(eff) }}$ is of first order) and given the symmetry in the solution, this is $\mathrm{d}^{2} x^{\perp}=2 \pi r \mathrm{~d} r$. The macroscopic stress-energy tensor $\widetilde{\mathbf{T}}_{\mathrm{M}}$, depending only on the internal string coordinates $\xi_{a}$, is derivable from the relation

$$
\widetilde{T}_{\|}^{\mu \nu}\left(x^{\alpha}\right)=\int \widetilde{T}_{\mathrm{M}}^{\mu \nu} \delta\left[x^{\alpha}-X^{\alpha}\left(\xi_{a}\right)\right] \mathrm{d}^{2} \xi,
$$

with $a=0,1$ and $X^{\alpha}\left(\xi_{a}\right)$ defining the two-parameter locus of the string. In the case at hand for which the string is aligned along the $z$ axis, the coordinates $\xi_{a}$ can be identified with $t$ and $z$; we shall make this choice in what follows.

Since one expects $\widetilde{T}_{\text {(eff) }}^{\mu \nu}$ to be conserved ${ }^{2}$ by virtue of Eq. (2), reproducing the steps of Ref. [11] leads to the fact that $\widetilde{\mathbf{T}}_{\mathrm{M}}$ can only depend on two integrated quantities. It turns out that it can in fact be given the form

$$
\widetilde{T}_{\mathrm{M}}=U u^{\mu} u^{\nu}-T v^{\mu} v^{\nu},
$$

with $\mathbf{u}$ and $\mathbf{v}$ respectively a unit timelike and spacelike vector parallel to the string worldsheet; again, in our case, these are $\mathbf{u}=(1,0,0,0)$ and $\mathbf{v}=(0,1,0,0)$ [recall Eq. (17)].

The eigenvalues of $\widetilde{\mathbf{T}}_{\mathrm{M}}$ are the energy per unit length $U$ and the tension $T$, which we are now in a position to express directly from the effective stress-energy tensor as

$$
U=2 \pi \int \widetilde{T}_{(\mathrm{eff})}^{t t} r \mathrm{~d} r
$$

and

$$
T=-2 \pi \int \widetilde{T}_{(\mathrm{eff})}^{z z} r \mathrm{~d} r
$$

Turning back to Eq. (55), it is straightforward to convince oneself that these quantities take the form

$$
\begin{gathered}
U=m_{0}^{2}+2 \pi \int \partial_{t}^{2} \widetilde{\Phi} r \mathrm{~d} r, \\
T=m_{0}^{2}-2 \pi \int \partial_{z}^{2} \widetilde{\Phi} r \mathrm{~d} r,
\end{gathered}
$$

where $m_{0}^{2}$ is an integral over the transverse direction of the part of the microscopic fields that depends only on the radial distance $r$, i.e. a constant with the dimension of a mass square (hence the notation).

Using the solutions (37) and (50) together with the expansion (54), we get

$$
U=m_{0}^{2}+4 \pi \alpha_{0} \omega^{2} \epsilon \psi(k z-\omega t) \int f(r) r \mathrm{~d} r
$$

and

$$
T=m_{0}^{2}-4 \pi \alpha_{0} k^{2} \epsilon \psi(k z-\omega t) \int f(r) r \mathrm{~d} r
$$

relations that imply that not only is the stress-energy tensor no longer degenerate when inclusion of the dilatonic field is taken into account, but also that the resulting string is not of

\footnotetext{
${ }^{2}$ Note in that respect that Eq. (3) can be seen as a simple consequence of Eqs. (2) and (4), i.e. of the conservation of the matter stress-energy tensor and that of Einstein tensor.
} 
the Witten superconducting kind $[13,15]$ since the energy per unit length and tension explicitly depend on the internal coordinates.

From Eqs. (62) and (63) and the solution (51), one sees immediately that because of the asymptotic behavior of the Bessel functions $J_{0}$ and $Y_{0}$, the timelike case $w<0$ is excluded for the case at hand since it leads to divergent integrals in $U$ and $T$. Indeed, the integrals (see Eq. (5.52/1) in Ref. [27]) are ${ }^{3}$ proportional to $\int r \mathcal{Z}_{0}(\omega r) \mathrm{d} r=(r /$ $\omega) \mathcal{Z}_{1}(\omega r)$, which asymptotically behaves as $\sqrt{r_{\infty}}$, with $r_{\infty}$ an appropriate cutoff, for the timelike case (51), and exponentially converges for the spacelike case (52).

The timelike case can however be accounted for by adding a potential term for the dilaton, i.e. by substituting the Einstein frame action (7) with

$$
\begin{aligned}
\mathcal{S}_{\mathrm{E}}^{(\mathrm{pot})}= & \frac{1}{16 \pi G^{*}} \int \mathrm{d}^{4} x \sqrt{-g}\left[R-2 g^{\mu \nu} \partial_{\mu} \phi \partial_{\nu} \phi+V(\phi)\right] \\
& +\mathcal{S}_{\mathrm{m}}\left[\Psi_{\mathrm{m}}, A^{2}(\phi) g_{\mu \nu}\right],
\end{aligned}
$$

which turns Eq. (12) into

$$
\square \phi=-\frac{1}{4} \frac{\mathrm{d} V}{\mathrm{~d} \phi}-4 \pi G^{*} \alpha(\phi) T .
$$

Defining the dilaton mass $M_{\mathrm{d}}$ through

$$
\left.M_{\mathrm{d}}^{2} \equiv \frac{1}{4} \frac{\mathrm{d}^{2} V}{\mathrm{~d} \phi^{2}}\right|_{\phi=\phi_{0}},
$$

and following the same steps as in Sec. IV, we find that the first order dilaton $\phi_{1}$ satisfies

$$
\left(\ddot{\phi}_{1}-\overline{\bar{\phi}}_{1}\right)-\frac{1}{r} \frac{\partial}{\partial r}\left(r \frac{\partial \phi_{1}}{\partial r}\right)+M_{\mathrm{d}}^{2} \phi_{1}=\mathcal{F}(r),
$$

instead of Eq. (36). To obtain Eq. (67), we have made use of the fact that in this context, $\phi_{0}$ is the vacuum expectation value of $\phi$ and the dilaton is stabilized in the sense that $\mathrm{d} V / \mathrm{d} \phi=0$ for $\phi=\phi_{0}$ and that $M_{\mathrm{d}}^{2}>0$. Making use again of the expansion (37) and choosing the purely radial part to satisfy

$$
\chi^{\prime \prime}+\frac{1}{r} \chi^{\prime}-M_{\mathrm{d}}^{2} \chi=\mathcal{F}(r)
$$

instead of Eq. (39), one sees that Eq. (45) is left unchanged, implying the same solution (50), while Eq. (46) is turned into

$$
f^{\prime \prime}+\frac{1}{r} f^{\prime}=\left(M_{\mathrm{d}}^{2}+w\right) f,
$$

whose solution is an exponentially convergent modified Bessel function [cf. Eq. (50)] provided the phase frequency, in the frame in which $k \rightarrow 0$, is below the threshold [15] $\omega$

\footnotetext{
${ }^{3}$ We call $\mathcal{Z}_{p}$ any Bessel function, modified or not, of order $p$.
}

$<\omega_{\mathrm{th}}=M_{\mathrm{d}}$. It is remarkable that this threshold is, just like in the Witten superconducting string model [13], also set by the mass of what one could thus, by analogy, call the current carrier.

From the point of view of cosmology, the stress-energy tensor eigenvalues given by Eqs. (62) and (63) depend on the internal worldsheet coordinates, but in a very special way. In fact, defining the average of the quantity $X$ over the spacelike or timelike variable $u=k z-\omega t$ by means of

$$
\langle X\rangle \equiv \frac{1}{2 \pi} \int_{-\pi}^{\pi} X(u) \mathrm{d} u,
$$

we obtain the very simple Nambu-Goto [20] relation

$$
\langle U\rangle=\langle T\rangle=m_{0}^{2},
$$

showing that on distances much larger than the characteristic dilaton length scale, for instance over distances of cosmological relevance, a network of such cosmic strings will evolve in a way that is similar to ordinary (non-currentcarrying) strings. In particular, one therefore expects the overall network to rapidly reach a scaling solution and to produce a large number of small loops [19]. At this level, all the cosmological predictions of the ordinary string models are unchanged.

Once we consider the smaller loops, however, the situation can be drastically modified, in a fashion comparable to the vorton case [16]: since the degeneracy between $U$ and $T$ is raised microscopically, one might also expect centrifugally supported equilibrium configurations to exist, leading to the usual vorton excess problem. Indeed, the presence of an explicit phase factor $\psi$ in their definition implies that the quantization condition in the spacelike case

$$
k L=2 \pi N,
$$

with $N \in \mathbb{N}$ and $L$ the loop circumference, must hold. It is not clear however if the dilaton can leave the string, and the mechanism by which it could be possible presumably depends on what happens below the cutoff at short distance.

Finally, one can note that the difference between the eigenvalues $U$ and $T$ is $U-T \propto \psi$, which, given Eq. (50), is not positive definite. This can be traced back to the well-known expected violation of the null energy condition (NEC) in such scalar-tensor theories. A similar violation allows cosmological solutions to have bouncing scale factors with ordinary matter components (fluids and/or scalar fields) [2,28]. In the string case under consideration here, this seemingly acausal violation of the NEC stems from the coupling of the dilaton field to the matter fields. The causality issue here stems from the fact that in the usual treatment of string perturbations [14], the velocity of transverse perturbations along the string is found to be $c_{\mathrm{T}}^{2} \equiv T / U$, which clearly exceeds unity if the NEC is violated. However bizarre it may sound, this need not worry us unduly. Indeed, since the theory given by Eq. (1) satisfies the requirement of causality however, it is clear that $c_{\mathrm{T}}^{2}$ cannot, in this framework, represent the propagation velocity of transverse perturbations along the string. 


\section{CONCLUSIONS}

We have reexamined the field equations of a cosmic string coupled to a tensor-scalar theory of gravity. This coupling is shown to induce effects along the string comparable to a current flow in the sense that the resulting effective stressenergy tensor eigenvalues, the energy per unit length $U$ and tension $T$, are no longer degenerate, due to the presence of the dilaton. However, we have found that there are many differences with the ordinary mechanism of current formation in cosmic strings as was first proposed by Witten [13]. In the latter case, it was shown [15] that the generated currents can be either of the spacelike kind or of the timelike kind. In the dilatonic situation under scrutiny here, the timelike case is found to be pathological and can be accounted for only by addition of a potential term for the dilaton. Once this is done however, one finds that the phase frequency threshold derived in the Witten case [15] has an exact counterpart since the timelike kind of "current" can only be reached provided the equivalent of the state parameter is larger than the dilaton mass.

Another difference concerns the fact that the current is not formed after the string forming phase transition but should instead appear exactly at the same time. This is due to the fact that the dilaton is not an ordinary scalar field but acts as a component of the gravitational interaction. From this peculiarity also stems the possibility for the surface stress-energy tensor to be NEC-violating, in the sense that its timelike eigenvalue is not necessarily larger than its spacelike eigenvalue, as should be the case in the more restrictive field theory based situation. As a result, we have found that the average values of the macroscopically relevant quantities are unaltered by inclusion of dilatonic effects: the strings, from the point of view of cosmology, are expected to form a Nambu-Goto-like network.

However, the network of strings here produced would suffer from the vorton excess problem [16] because of the short- range effects of the dilaton. As the stress-energy tensor eigenvalues depend explicitly on the string worldsheet coordinates in a periodic way, it is necessary, in order to form a string loop, that the wave number of the dilatonic perturbation along the string be quantized.

We also found that even though a short-distance cutoff must be imposed, just like in the axionic situation [24], the long-distance behavior of the dilaton can be adjusted so as to suppress the logarithmic divergence. This means that a dilatonic network of strings, apart from having an energy per unit length that should be renormalized by inclusion of Planck scale effects, behaves exactly as a usual network. Accordingly, all the results derived for the latter [19] may be applied straightforwardly to the former, in particular in regards of the predictions relative to the loop production and the would-be "vorton" production.

More work needs to be done on such strings, as it is not clear in particular whether there is any way to get rid of the "current." If no possibility can be found, that would mean that the vortons-like configurations produced after the relevant phase transition would be absolutely stable. As a result, one would have to conclude that cosmic strings cannot be formed in the early universe if the underlying theory of gravity is of the scalar-tensor type. Such models, stemming from string theory, would therefore be incompatible with cosmic strings; in view of the recently released CMB data [18], this might be presented as a useful constraint for the underlying microscopic field theory.

\section{ACKNOWLEDGMENTS}

V.C. de A. would like to thank FAPESP - BRASIL for financial support. M.E.X.G. would like to acknowledge the kind hospitality of the Institut d'Astrophysique de Paris where this work has been completed. We would also like to thank Jérôme Martin and Raymond Schutz for enlightening comments and discussions.
[1] M. B. Green, J. H. Schwarz, and E. Witten, Superstring Theory (Cambridge University Press, Cambridge, England, 1987); J. Polchinski, String Theory (Cambridge University Press, Cambridge, England, 1998).

[2] J.C. Fabris, R.G. Furtado, P. Peter, and N. Pinto-Neto, this issue, Phys. Rev. D 67, 124003 (2003).

[3] C. Brans and R.H. Dicke, Phys. Rev. 24, 925 (1961); P.G. Bermann, Int. J. Theor. Phys. 1, 25 (1968); R.V. Wagoner, Phys. Rev. D 1, 3209 (1970); K. Nordtvedt, Astrophys. J. 161, 1059 (1970).

[4] D. La and P.J. Steinhardt, Phys. Rev. Lett. 62, 376 (1989); P.J. Steinhardt and F.S. Accetta, ibid. 64, 2740 (1990).

[5] T.W.B. Kibble, J. Phys. A 9, 1387 (1976); Phys. Rep. 67, 183 (1980); E. P. S. Shellard and A. Vilenkin, Cosmic Strings and Other Topological Defects (Cambridge University Press, Cambridge, England, 1994); M.B. Hindmarsh and T.W.B. Kibble, Rep. Prog. Phys. 58, 477 (1995); Formation and Interactions of Topological Defects, Vol. 49 of NATO Advanced Study Institute Series B: Physics, edited by R. Brandenberger and A.-C.
Davis (Plenum, New York, 1995).

[6] M.E.X. Guimarães, Class. Quantum Grav. 14, 435 (1997).

[7] T. Damour and A.M. Polyakov, Nucl. Phys. B423, 532 (1994).

[8] T. Damour and K. Nordtvedt, Phys. Rev. Lett. 70, 2217 (1993); Phys. Rev. D 48, 3436 (1993).

[9] V.A. Ruban and A.M. Finkelstein, Lett. Nuovo Cimento Soc. Ital. Fis. 5, 289 (1972).

[10] T. Vachaspati, Phys. Rev. D 45, 3487 (1992).

[11] P. Peter, Class. Quantum Grav. 11, 131 (1994).

[12] C.N. Ferreira, M.E.X. Guimarães, and J.A. Helayël-Neto, Nucl. Phys. B581, 165 (2000).

[13] E. Witten, Nucl. Phys. B249, 557 (1985).

[14] B. Carter, Phys. Lett. B 224, 61 (1989); 228, 466 (1989); Nucl. Phys. B412, 345 (1994); see also B. Carter, P. Peter, and A. Gangui, Phys. Rev. D 55, 4647 (1997).

[15] P. Peter, Phys. Rev. D 45, 1091 (1992); 46, 3335 (1992).

[16] R.L. Davis Phys. Rev. D 38, 3722 (1988); R.L. Davis and E.P.S. Shellard, Nucl. Phys. B323, 209 (1989); B. Carter, Ann. N.Y. Acad. Sci. 647, 758 (1991); B. Carter, Phys. Lett. B 238, 
166 (1990); in Proceedings of the XXXth Rencontres de Moriond, Villard-sur-Ollon, Switzerland, 1995, edited by B. Guiderdoni and J. Tran Thanh Vân (Editions Frontières, Gif-surYvette, 1995); R. Brandenberger, B. Carter, A.C. Davis, and M. Trodden, Phys. Rev. D 54, 6059 (1996); B. Carter and A.C. Davis, ibid. 61, 123501 (2000).

[17] A. Riazuelo, N. Deruelle, and P. Peter, Phys. Rev. D 61, 123504 (2000).

[18] C.L. Bennett et al., astro-ph/0302207; D.N. Spergel et al., astro-ph/0302209.

[19] D. Austin, E. Copeland, and T.W.B. Kibble, Phys. Rev. D 48, 5594 (1993); A. Albrecht and N. Turok, Phys. Rev. Lett. 54, 1868 (1985); Phys. Rev. D 40, 973 (1989); D.P. Bennett and F.R. Bouchet, Phys. Rev. Lett. 60, 257 (1988); 63, 2776 (1989); Phys. Rev. D 41, 2408 (1990); B. Allen and E.P.S. Shellard, Phys. Rev. Lett. 64, 119 (1990).

[20] T. Goto, Prog. Theor. Phys. 46, 1560 (1971); Y. Nambu, Phys.
Rev. D 10, 4262 (1974).

[21] T. Damour, Nucl. Phys. B (Proc. Suppl.) 80, 41 (2000).

[22] H.B. Nielsen and P. Olesen, Nucl. Phys. B61, 45 (1973).

[23] F.R. Bouchet, P. Peter, A. Riazuelo, and M. Sakellariadou, Phys. Rev. D 65, 021301(R) (2002).

[24] P. Binétruy, C. Deffayet, and P. Peter, Phys. Lett. B 441, 52 (1998).

[25] M. Dine, N. Seiberg, and E. Witten, Nucl. Phys. B289, 589 (1987); J. Atick, L. Dixon, and A. Sen, ibid. B292, 109 (1987); M. Dine, I. Ichinose, and N. Seiberg, ibid. B293, 253 (1988).

[26] A. L. N. Oliveira and M. E. X. Guimarães, Phys. Lett. A 311, 474 (2003).

[27] I. S. Gradshteyn and I. M. Ryshik, Table of Integrals, Series and Products (Academic Press, New York, 1980).

[28] C. Molina-Paris and M. Visser, Phys. Lett. B 455, 90 (1999); see also P. Peter and N. Pinto-Neto, Phys. Rev. D 65, 023513 (2002), and references therein. 